\title{
Modeling of the overwintering distribution of Puccinia strifformis f. sp. tritici based on meteorological data from 2001 to 2012 in China
}

\author{
Xiaojing WANG ${ }^{1}$, Zhanhong MA ${ }^{1}$, Yuying JIANG ${ }^{2}$, Shouding SHI ${ }^{3}$, Wancai LIU ${ }^{2}$, Juan ZENG ${ }^{2}$, Zhiwei ZHAO ${ }^{1}$, \\ Haiguang WANG (凶) ${ }^{1}$
}

1 College of Agriculture and Biotechnology, China Agricultural University, Beijing 100193, China

2 National Agro-Tech Extension and Service Center, Ministry of Agriculture, Beijing 100125, China

3 China Animal Agriculture Association, Beijing 100028, China

\begin{abstract}
Wheat stripe rust caused by Puccinia striiformis f. sp. tritici occurs widely in China and seriously affects wheat production. Global warming could profoundly impact the incidence and prevalence of lowtemperature diseases such as stripe rust. Studies on the effects of temperature on the distribution of overwintering stripe rust could help us understand the incidence and prevalence of the disease and could also provide support for monitoring, forecasting and developing control strategies. An exponential model and a spherical model of the ordinary Kriging method in the ArcGIS platform were used to predict the overwintering regions of stripe rust based on the probability that the average temperature of the coldest month from December to February was higher than -6 or $-7^{\circ} \mathrm{C}$ from 2001 to 2012 . The results showed that the areas with a probability between $70 \%$ and $90 \%$ were transition regions for the overwintering of stripe rust. Based on annual mean temperature of the coldest month from December to February for 2001 to 2012, overwintering distribution of stripe rust was likewise evaluated. The boundary for overwintering of stripe rust was consistent with the areas where the probability was predicted to be $70 \%$ to $90 \%$ for the overwintering distribution of stripe rust, but the boundary was shifted northward toward Beijing in North China. Some areas in Xinjiang, including Akto, Pishan, Hotan and Yutian, were also predicted to be suitable for the overwintering of stripe rust.
\end{abstract}

Keywords stripe rust, wheat, overwintering, geospatial distribution, geographic information system, climate change

Received September 5, 2014; accepted October 9, 2014

Correspondence: wanghaiguang@cau.edu.cn

\section{Introduction}

Stripe (yellow) rust caused by Puccinia striiformis f. sp. tritici (Pst) is the most important disease of wheat in China. This disease occurs widely and often causes epidemics in wheat growing areas. It seriously affects the wheat production in the north-western, northern, south-western and other regions of China [1]. Wheat yield can be reduced by $10 \%$ to $30 \%$ by stripe rust, and total yield loss can occur when the disease is severe [1]. Since the 1950s, epidemics of stripe rust have occurred many times in China, seriously affecting wheat production. For instance, the pandemics of the disease in 1950, 1964, 1990 and 2002 caused wheat yield losses as high as 6.0, 3.2, 1.8 and 1.3 million tons, respectively [1,2]. Understanding the forces that drive stripe rust epidemics is important so that we can predict the disease, develop control strategies and implement timely measures to protect wheat production and ensure food security in China.

China has the largest epidemics of stripe rust in the world and an independent pandemic system has been formed in China [1,3]. Three epidemiological regions including (i) northern China-north-western China-the middle and lower reaches of the Yangtze River, (ii) Xinjiang and (iii) Yunnan, have been identified in the pandemic system [1]. Due to the complex terrain and various ecological environmental conditions of China, Pst generally relies on the dispersal of urediniospores by airflow to complete its life cycle. The different functional areas, including overwintering areas, oversummering areas, bridge areas and epidemic areas, are involved in the completion of its life cycle. The interrelationships between the inoculum source areas are also very complicated [4]. The critical low temperature for Pst overwintering is -6 to $-7^{\circ} \mathrm{C}$ in the coldest months. 
However, if the wheat seedlings in the field are covered by snow, Pst can overwinter safely, even when the air temperature is lower than $-10^{\circ} \mathrm{C}$. Generally, the northern geographic boundary for Pst overwintering is a line from Dezhou (Shandong) through Shijiazhuang (Hebei) and Jiexiu (Shanxi) to Huangling (Shaanxi) [1]. The overwintering rate of Pst to the north of that line is very low.

In the Jianghuai Region, the Jianghan Region and the Sichuan Basin, however, the warm and wet winters allow Pst to continue to spread and infect wheat seedlings. These areas become the inoculum source for the wheat growing areas in northern China and the inocula from these areas can infect wheat seedlings in northern China in the following year. The number of $P s t$ inocula that overwinter has a significant influence on the spring epidemics of stripe rust and these epidemics are also influenced by weather conditions, so that meteorological factors such as temperature, humidity and rainfall can have significant effects on epidemics of stripe rust.

Climate change from global warming over the past one hundred years has attracted attention from governments and the public around the world. The Fourth Assessment Report issued by the Intergovernmental Panel on Climate Change (IPCC) in 2007 reported that the global average surface temperature increased by $0.74^{\circ} \mathrm{C}$ during the period 1906 to 2005 . This temperature is significantly higher than the $0.6^{\circ} \mathrm{C}$ temperature increase estimated for the period 1901 to 2001, as reported in the IPCC Third Assessment Report of 2001. As a result of global warming, the 100year average temperature in China has also increased by 0.5 to $0.8^{\circ} \mathrm{C}$. In the past 50 years, the increase in the annual average temperature in China has resulted mainly from an increase in the temperature in northern China [5]. The rate of temperature increase reached $0.8^{\circ} \mathrm{C}$ per 10 years in north-eastern China, Inner Mongolia and the western basin, and the temperature has increased more than $4^{\circ} \mathrm{C}$ in the past 50 years [5]. The effects of global climate change, particularly climate warming, on plant diseases have become a matter of widespread concern [6-9]. Climate warming will have effects on agriculture and agricultural pests in China [10-12] and studies are therefore needed on the effects of climate warming on stripe rust $[13,14]$.

An understanding of the natural laws that govern plant disease epidemics on the macroscale is important for the sustainable management of the diseases and macro decision-making. Geographic information systems (GIS) and geostatistics are important approaches and methods for studying the regional occurrence and epidemics of plant diseases [15]. Jaime-Garcia et al. [16] used geostatistics and GIS to investigate the spatial pattern of Phytophthora infestans genotypes in the Del Fuerte Valley, Sinaloa, Mexico. Using GIS that was based on the temperature and precipitation during the blossom period, Chen et al. [17] conducted a risk analysis to investigate the potential distribution of Erwinia amylovora (the causal agent of fire blight) in China, and the results showed that the pathogen could survive in most of China and that suitable host plants and environmental conditions could result in an epidemic of fire blight. Li et al. [18] used GIS to identify the oversummering regions of Blumeria graminis f. sp. tritici in China. Shi [19] modeled the oversummering distribution of Pst using ArcGIS 8.3 for two contexts. The first used the ordinary Kriging method based on the annual probability of the average temperature of the two warmest 10 days in July and August being below $20,21,22$ or $23^{\circ} \mathrm{C}$ during the years 1960 to 2001, and the predicted distribution fitted well with the actual situation as the critical temperature was $22^{\circ} \mathrm{C}$. The second used the ordinary Kriging method and the digital elevation model based on the average temperature of the warmest 10 days in July and August. Similarly, Shi [19] modeled the overwintering distribution of Pst. Using the annual probability that the average temperature of the coldest month was higher than $-5,-6$, $-7,-9$ or $-10^{\circ} \mathrm{C}$ during the years 1960 to 2001 , the model predicted that $-7^{\circ} \mathrm{C}$ was the optimal critical temperature for overwintering. The ordinary Kriging method in combination with the digital elevation model was also used to predict the overwintering distribution of Pst based on the average temperature of the coldest month selected from December in the previous year and the following January.

To investigate the effects of climate warming on epidemics of stripe rust in China and to provide support for monitoring, forecasting and controlling the disease, modeling of overwintering of Pst was conducted using ArcGIS10.0 based on meteorological data from December 2001 to February 2012. The results are expected to be useful in further determining the overwintering boundaries and predicting the impact of temperature change on overwintering of Pst in China. The information gained will be useful for correctly understanding the occurrence, forecast and control of stripe rust in China in the future.

\section{Data collection}

\subsection{Spatial data}

A provincial level administrative map showing province information, a county level administrative map showing county information and a map of China with digital elevation data were from the National Basic Geographic Information Center of China (http://nfgis.nsdi.gov.cn). All these maps used China Geodetic Coordinate System 2000 as the geographic coordinate system. A projection transformation with Hong Kong 1980 UTM Zone 49N as the projection coordinate system was applied to allow plane coordinates to be used for analysis. 


\subsection{Meteorological data}

The meteorological data used were from the China ground climate data monthly data set of the China Meteorological Data Sharing Service System (http://cdc.cma.gov.cn). The meteorological data from 884 meteorological stations included monthly average temperatures from December 2001 to February 2012. The station information of 731 meteorological stations was obtained from the information of 756 meteorological stations from the China Meteorological Data Sharing Service System. Then 41 stations with less than 5 years of data were excluded. The data of the 690 remaining meteorological stations as shown in Fig. 1, including monthly average temperature, longitude, latitude and elevation, were used in this study.

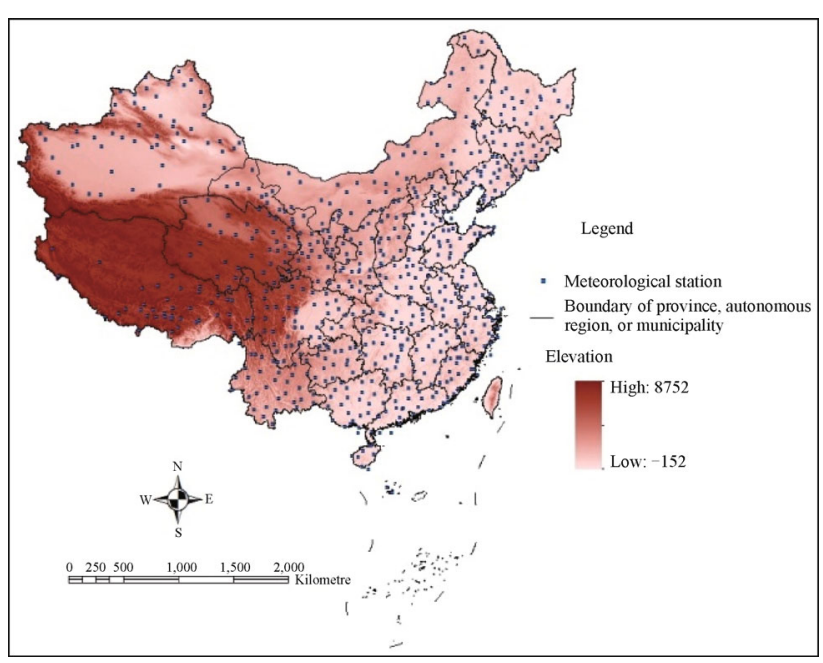

Fig. 1 Meteorological stations used for the modeling of overwintering distribution of Puccinia striiformis f. sp. tritici in China

2.3 Growing areas of winter wheat and historical overwintering locations of stripe rust

A wheat growing area map was drawn using the National Agricultural Atlas of the People's Republic of China [20]. A map of wheat ecological regions of China was obtained by digitizing a previously published map [21] using the software ArcGIS. The host growing areas included those of winter wheat only and of both winter and spring wheat, because only winter wheat is considered as the overwintering host. Areas only producing spring wheat were excluded. The map of the host growing areas (Fig. 2) was derived from the above two maps. The historical overwintering locations of stripe rust were obtained from a review of the literature. In total, 52 locations were found (Fig. 2), including 19 from Gansu, eight from Xinjiang, two from Tibet, one from Ningxia, five from Shaanxi, one from Shanxi, one from Henan, 14 from Sichuan, and one from Yunnan.

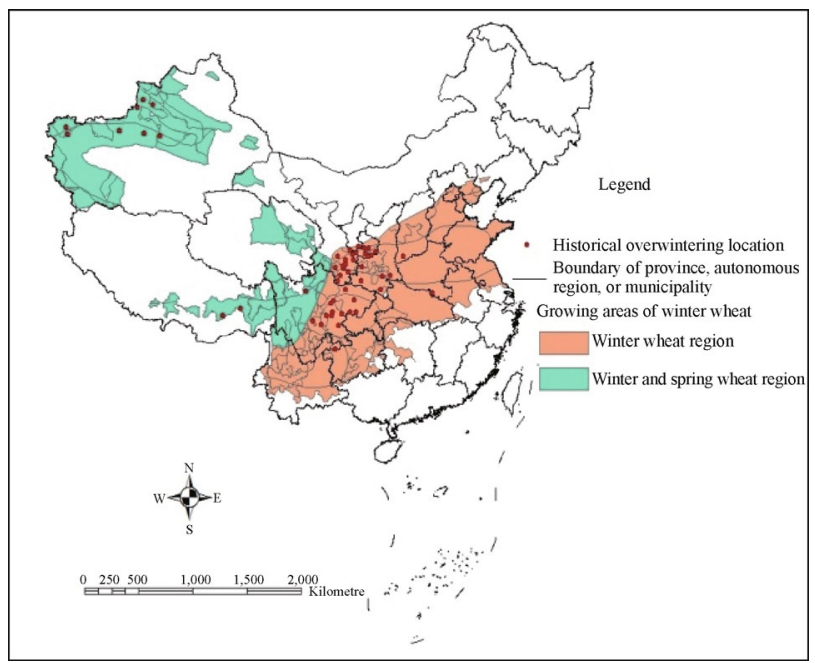

Fig. 2 Growing areas of winter wheat and selected historical overwintering locations of stripe rust in China

\section{Modeling of overwintering distribution}

3.1 Modeling of the overwintering distribution of Pst based on the probability that the average temperature of the coldest month was higher than the critical temperature

\subsubsection{Distribution indices}

The probability that the average temperature of the coldest month (from December, January and February) for the period from December 2001 to February 2012 at each meteorological station was higher than the critical temperature $\left(-6\right.$ or $\left.-7^{\circ} \mathrm{C}\right)$ was treated as the main distribution index for overwintering of Pst based on the temperature data. The host of Pst was treated as another main distribution index for the overwintering of Pst. Only winter wheat was treated as the host for Pst overwintering, so the host growing areas included this wheat growing region and the winter and spring wheat growing region.

\subsubsection{Data processing}

The average temperature of the coldest month of December, January or February from each meteorological station was calculated. Then, the probability that the average temperature of the coldest month was higher than the critical temperature of each station was calculated using relevant functions, CUNTIFS and COUNTIF.

\subsubsection{Modeling method}

Geostatistical analysis was performed using ArcGIS 10.0 and, based on the fit, five relatively good interpolation methods were selected, including Inverse Distance Weighting (IDW), Global Polynomial Interpolation 
(GPI), Local Polynomial Interpolation (LPI), Radial Basis Functions (RBF) and Kriging. The optimal method was obtained according to interpolation evaluation of the five methods.

When IDW was used, the interpolation effects were controlled mainly by adjusting the power values. There were more mistakes in the results with an increase in the power value. In this study, the power was set as 1,2, 3 and 4. The power with highest regression coefficient $(\mathrm{R})$, the minimal mean as close to 0 as possible, and the lowest root mean square (RMS), was treated as the optimal power. When GPI was used, the interpolation effects were controlled by adjusting the Order of Polynomial (OP) similar to the power of IDW, and the evaluation standard was also similar to IDW. The evaluation standard of LPI was also similar to IDW, and OP was used to control the interpolation effects. For LPI, the recommended orders are generally 1 to 3 , so a value greater than 3 was not used in this study. The interpolation effects of LPI were also affected by kernel functions including Exponential (Ex), Polynomial Order 5 (PO5), Gaussian (G), Epanechnikov (Ep), Quartic (Q) and Constant. The evaluation standard of RBF was similar to IDW and LPI, and the interpolation effects were affected by five functions including the Completely Regularized Spline (CRS), Spline With Tension (SWT), Multiquadric Functions (MF), Inverse Multiquadric Spline (IM) and Thin-Plate Spline (TPS).

Ordinary Kriging (OK) is a type of geostatistics method that includes spatial autocorrelation analysis. The ratio of nugget value and sill value $(\mathrm{C} /)$ can reflect the spatial correlation. The ordinary Kriging interpolation method can use semivariogram and covariance for modeling. In this study, when the covariance approach was used to build a model, a higher $\mathrm{C} /$ was obtained, and this result indicated that the spatial correlation was weaker. Therefore, a semivariogram approach was used for modeling in this study. The model with a higher R, a mean standardized (MS) value as close to 0 as possible, the least RMS and average standard error (ASE), and a root mean square standardized (RMSS) value as close to 1 as possible was selected as the optimal model for overwintering distribution of Pst. The interpolation effects of an ordinary Kriging interpolation method were also affected by the model type, including Circular (C), Spherical (Sp), Tetraspherical (Tsp), Pentaspherical (Psp), Exponential (E), Gaussian (G), Rational Quadratic (RQ), Hole Effect (HF), K-Bessel (K-B), J-Bessel (J-B), and Stable (St).

3.2 Modeling of the overwintering distribution of Pst based on the annual mean temperature of the coldest month and elevation model

\subsubsection{Distribution index}

The annual average of the temperature of the coldest month from December, January and February in each year, for the period from December 2001 to February 2012 (the annual mean temperature of the coldest month in abbreviation) was treated as the distribution index for overwintering of Pst.

\subsubsection{Modeling method}

The provincial level administrative map and the map of the winter wheat growing areas were treated as base maps. Modeling of the overwintering distribution of Pst was conducted using ArcGIS 10.0 in combination with the elevation model of China. The optimal interpolation method determined above was applied in this analysis. Considering the effects of altitude on temperature, temperature conversion was conducted before interpolation. In other words, using the relationship between temperature and altitude, we transformed the temperature of each meteorological station into the temperatures where the altitude was $0 \mathrm{~m}$, and then the interpolation was conducted. An accurate temperature distribution for winter wheat growing areas was obtained with grid computing in combination with an elevation map of China. Then, the temperatures were compared with the critical temperature, and finally, the range of overwintering distribution was obtained. In this study, two methods were used to determine the relation between temperature and altitude. The first method used the standard relationship to describe the relation between temperature and altitude. The equation was $t_{0}=t+0.006 \times A$, for which $t_{0}$ was the temperature where the altitude was $0 \mathrm{~m}, t$ was the annual mean temperature of the coldest month of the meteorological station and $A$ was the altitude of the meteorological station. The second was the regression analysis method to build the equation between the annual mean temperature of the coldest month and the altitude of the corresponding meteorological station. The equation obtained was $t_{0}=t+0.00159 \times A$, for which the definition of the parameters was the same as those in the standard equation. The equation showed that the temperature decreased $1.59^{\circ} \mathrm{C}$ as the altitude rose $1000 \mathrm{~m}$.

\section{Results}

4.1 Overwintering distribution predictions based on the probability that the average temperature of the coldest month was higher than the critical temperature

\subsubsection{Evaluation using IDW as the interpolation method}

The evaluation parameters after interpolation for the different powers of IDW are shown in Table 1. With an increase in power, $\mathrm{R}$ gradually increased, and the mean and RMS showed a somewhat increasing trend. Considering $\mathrm{R}$, 
the mean and RMS, the optimal power was determined. Because all values of $\mathrm{R}$ were high, power 1 , which gave a small mean and RMS, was chosen as the optimal power in order to minimize the prediction error.

\subsubsection{Evaluation using GPI as the interpolation method}

The evaluation parameters after interpolation with different orders of GPI are shown in Table 2. When the order of GPI was 3, the maximum of $\mathrm{R}$ was obtained, and the mean and RMS were relatively small. Therefore, 3 was chosen as the optimal order of GPI.

\subsubsection{Evaluation using LPI as the interpolation method}

The evaluation parameters after interpolation with different orders and functions of LPI are shown in Table 3. As the order was 1 and the kernel function was constant, the minimum values of RMS were both obtained when the interpolation was conducted based on the probability that the average temperature of the coldest month was higher than the critical temperature $\left(-6\right.$ or $\left.-7^{\circ} \mathrm{C}\right)$. At the same time, because the means were close to 0 , the regression coefficients were relatively high. Therefore, 1 was taken as the order, and the constant function was treated as the kernel function for interpolation.

4.1.4 Evaluation results using $\mathrm{RBF}$ as the interpolation method

The evaluation parameters after interpolation with different functions of RBF are shown in Table 4. When MF was used for interpolation, the minimum values of RMS were both obtained, the means were close to 0 , and the regression coefficients were relatively high. Therefore, MF was chosen as the function of RBF for interpolation.

\subsubsection{Evaluation using Kriging as the interpolation method}

The evaluation parameters after interpolation with different models of Kriging are shown in Table 5. When the model of Kriging, which is an exponential model, was used, the maximum regression coefficient, the minimum ASE and the minimum $\mathrm{C} / \mathrm{were}$ obtained. Although all best evaluation standards cannot be met, the exponential model was relatively good. Therefore, the exponential model was chosen as the optimal model for interpolation.

\subsubsection{Comprehensive evaluation results}

A comparison of the evaluation parameters with the optimal results for each interpolation method was conducted, and the best interpolation method was then selected. Among the five interpolation methods, IDW, GPI, LPI and RBF only had three evaluation parameters, namely, R, the mean and RMS. Therefore, the same three evaluation parameters were also used to evaluate the Kriging method. As shown in Table 6, the ordinary Kriging interpolation method was better. Therefore, the Kriging method with semivariogram function and exponential model was selected as the interpolation method for modeling the overwintering distribution of Pst. Moreover, in order to compare our results with those of Shi [19], the Kriging method with semivariogram function and spherical model was also used. The evaluation parameters shown in Table 5 indicate that the spherical model was relatively good.

Table 1 Comparison of evaluation parameters after interpolation with different powers of IDW

\begin{tabular}{|c|c|c|c|c|c|c|}
\hline \multirow{2}{*}{ Power } & \multicolumn{3}{|c|}{$P>-6^{\circ} \mathrm{C}$} & \multicolumn{3}{|c|}{$P>-7^{\circ} \mathrm{C}$} \\
\hline & $\mathrm{R}$ & Mean & RMS & $\mathrm{R}$ & Mean & RMS \\
\hline 1 & 0.9291 & 0.0059 & 0.1568 & 0.9164 & 0.0071 & 0.1612 \\
\hline 2 & 0.9395 & 0.0066 & 0.1564 & 0.9276 & 0.0082 & 0.1616 \\
\hline 3 & 0.9471 & 0.0061 & 0.1604 & 0.9350 & 0.0082 & 0.1661 \\
\hline 4 & 0.9525 & 0.0053 & 0.1654 & 0.9400 & 0.0078 & 0.1713 \\
\hline
\end{tabular}

Note: $P>-6^{\circ} \mathrm{C}$ means the probability that the average temperature of the coldest month from December to February was higher than $-6^{\circ} \mathrm{C}$ from 2001 to 2012 ; $P>-7^{\circ} \mathrm{C}$ means the probability that the average temperature of the coldest month from December to February was higher than $-7^{\circ} \mathrm{C}$ from 2001 to 2012 .

Table 2 Comparison of evaluation parameters after interpolation with different orders of GPI

\begin{tabular}{|c|c|c|c|c|c|c|}
\hline \multirow{2}{*}{$\mathrm{OP}$} & \multicolumn{3}{|c|}{$P>-6^{\circ} \mathrm{C}$} & \multicolumn{3}{|c|}{$P>-7^{\circ} \mathrm{C}$} \\
\hline & $\mathrm{R}$ & Mean & RMS & $\mathrm{R}$ & Mean & RMS \\
\hline 1 & 0.6042 & 0.00002 & 0.2798 & 0.6177 & 0.00003 & 0.2802 \\
\hline 2 & 0.6141 & -0.0001 & 0.2716 & 0.6247 & -0.0001 & 0.2697 \\
\hline 3 & 0.8357 & -0.0001 & 0.2323 & 0.8271 & -0.0001 & 0.2347 \\
\hline 4 & 0.8226 & 0.0002 & 0.2196 & 0.8224 & 0.0002 & 0.2196 \\
\hline
\end{tabular}

Note: $P>-6^{\circ} \mathrm{C}$ or $P>-7^{\circ} \mathrm{C}$ has the same mean as Table 1 . 
Table 3 Comparison of evaluation parameters after interpolation with different orders and functions of LPI

\begin{tabular}{|c|c|c|c|c|c|c|c|}
\hline \multirow{2}{*}{$\mathrm{OP}$} & \multirow{2}{*}{ Kernel function } & \multicolumn{3}{|c|}{$P>-6^{\circ} \mathrm{C}$} & \multicolumn{3}{|c|}{$P>-7^{\circ} \mathrm{C}$} \\
\hline & & $\mathrm{R}$ & Mean & RMS & $\mathrm{R}$ & Mean & RMS \\
\hline \multirow{6}{*}{1} & Ex & 0.9470 & 0.0009 & 0.1496 & 0.9387 & 0.0019 & 0.1531 \\
\hline & PO5 & 0.8978 & -0.0015 & 0.1639 & 0.8854 & -0.0029 & 0.1668 \\
\hline & G & 0.9210 & 0.0001 & 0.1552 & 0.9069 & -0.0004 & 0.1588 \\
\hline & Ep & 0.8701 & -0.0041 & 0.1770 & 0.8584 & -0.0038 & 0.1828 \\
\hline & Q & 0.8791 & 0.0019 & 0.1727 & 0.8696 & -0.0035 & 0.1755 \\
\hline & Constant & 0.9394 & -0.0004 & 0.1487 & 0.9302 & -0.0007 & 0.1528 \\
\hline \multirow{6}{*}{2} & Ex & 0.9383 & 0.0002 & 0.1563 & 0.9472 & 0.0009 & 0.1577 \\
\hline & PO5 & 0.9288 & 0.0004 & 0.1601 & 0.9239 & 0.0023 & 0.1647 \\
\hline & G & 0.9502 & -0.0007 & 0.1549 & 0.9561 & -0.0004 & 0.1562 \\
\hline & Ep & 0.9193 & 0.0004 & 0.1665 & 0.9079 & 0.0013 & 0.1702 \\
\hline & Q & 0.9374 & 0.0173 & 0.4776 & 0.9161 & 0.0014 & 0.1644 \\
\hline & Constant & 0.9498 & 0.0001 & 0.1668 & 0.9454 & 0.0011 & 0.1624 \\
\hline \multirow{6}{*}{3} & Ex & 0.9127 & 0.0001 & 0.1683 & 0.8818 & -0.0024 & 0.1784 \\
\hline & PO5 & 0.8951 & -0.0010 & 0.1788 & 0.8965 & 0.0005 & 0.1791 \\
\hline & G & 0.9074 & 0.0001 & 0.1715 & 0.9033 & 0.0004 & 0.1719 \\
\hline & Ep & 0.8853 & -0.0010 & 0.1841 & 0.8856 & -0.0007 & 0.1842 \\
\hline & Q & 0.8966 & -0.0002 & 0.1803 & 0.8926 & 0.0001 & 0.1801 \\
\hline & Constant & 0.8655 & -0.0025 & 0.1919 & 0.8615 & -0.0025 & 0.1932 \\
\hline
\end{tabular}

Note: $P>-6^{\circ} \mathrm{C}$ or $P>-7^{\circ} \mathrm{C}$ has the same mean as Table 1 .

Table 4 Comparison of evaluation parameters after interpolation with different functions of RBF

\begin{tabular}{|c|c|c|c|c|c|c|}
\hline \multirow{2}{*}{ Function } & \multicolumn{3}{|c|}{$P>-6^{\circ} \mathrm{C}$} & \multicolumn{3}{|c|}{$P>-7^{\circ} \mathrm{C}$} \\
\hline & $\mathrm{R}$ & Mean & RMS & $\mathrm{R}$ & Mean & RMS \\
\hline CRS & 0.9705 & 0.0012 & 0.1727 & 0.9562 & 0.0031 & 0.1780 \\
\hline SWT & 0.9769 & 0.0011 & 0.1593 & 0.9614 & 0.0026 & 0.1675 \\
\hline MF & 0.9663 & 0.0015 & 0.1515 & 0.9521 & 0.0024 & 0.1578 \\
\hline IM & 0.9745 & 0.0007 & 0.1775 & 0.9595 & 0.0030 & 01852 \\
\hline TPS & 0.9825 & 0.0003 & 0.1627 & 0.9695 & 0.0022 & 0.1720 \\
\hline
\end{tabular}

Note: $P>-6^{\circ} \mathrm{C}$ or $P>-7^{\circ} \mathrm{C}$ has the same mean as Table 1 .

Table 5 Comparison of evaluation parameters after interpolation with different Kriging models

\begin{tabular}{|c|c|c|c|c|c|c|c|c|c|c|c|c|}
\hline \multirow{2}{*}{ Model } & \multicolumn{6}{|c|}{$P>-6^{\circ} \mathrm{C}$} & \multicolumn{6}{|c|}{$P>-7^{\circ} \mathrm{C}$} \\
\hline & $\mathrm{R}$ & MS & RMS & ASE & RMSS & $\mathrm{C} /(\%)$ & $\mathrm{R}$ & MS & RMS & ASE & RMSS & $\mathrm{C} /(\%)$ \\
\hline $\mathrm{C}$ & 0.9639 & 0.0049 & 0.1492 & 0.1252 & 0.1987 & 0.70 & 0.9534 & 0.0056 & 0.1538 & 0.1320 & 1.1604 & 1.29 \\
\hline $\mathrm{Sp}$ & 0.9646 & 0.0049 & 0.1496 & 0.1220 & 1.2398 & 0.45 & 0.9541 & 0.0059 & 0.1542 & 0.1294 & 1.1888 & 1.05 \\
\hline $\mathrm{TSp}$ & 0.9650 & 0.0050 & 0.1498 & 0.1212 & 1.2500 & 0.39 & 0.9545 & 0.0060 & 0.1544 & 0.1285 & 1.1998 & 0.94 \\
\hline $\mathrm{PSp}$ & 0.9670 & 0.0049 & 0.1509 & 0.1150 & 1.3412 & 0 & 0.9545 & 0.0064 & 0.1549 & 0.1250 & 1.2419 & 6.94 \\
\hline $\mathrm{E}$ & 0.9666 & 0.0047 & 0.1509 & 0.1407 & 1.0954 & 0 & 0.9579 & 0.0071 & 0.1572 & 0.1368 & 1.1679 & 0 \\
\hline G & 0.9099 & -0.0045 & 0.1571 & 0.1891 & 0.8279 & 9.72 & 0.9014 & -0.0062 & 0.1607 & 0.1912 & 0.8372 & 10.24 \\
\hline RQ & 0.9378 & -0.0104 & 0.1478 & 0.1388 & 1.0609 & 5.14 & 0.9266 & -0.0142 & 0.1526 & 0.1451 & 1.0469 & 5.80 \\
\hline $\mathrm{HF}$ & 0.9071 & -0.0025 & 0.1594 & 0.2065 & 0.7692 & 13.70 & 0.8986 & -0.0040 & 0.1627 & 0.2064 & 0.7855 & 14.14 \\
\hline K-B & 0.9232 & -0.0062 & 0.1520 & 0.1715 & 0.8832 & 6.92 & 0.9071 & -0.0077 & 0.1587 & 0.1825 & 0.8665 & 8.70 \\
\hline $\mathrm{J}-\mathrm{B}$ & 0.9082 & -0.0040 & 0.1576 & 0.1933 & 0.8124 & 10.36 & 0.9005 & -0.0054 & 0.1613 & 0.1970 & 0.8160 & 11.03 \\
\hline $\mathrm{St}$ & 0.9289 & -0.0042 & 0.1502 & 0.1707 & 0.8752 & 6.84 & 0.9195 & -0.0062 & 0.1550 & 0.1760 & 0.8755 & 7.64 \\
\hline
\end{tabular}

Note: $P>-6^{\circ} \mathrm{C}$ or $P>-7^{\circ} \mathrm{C}$ has the same mean as Table 1 . 
Table 6 Comparison of evaluation parameters after five interpolation methods

\begin{tabular}{|c|c|c|c|c|c|c|}
\hline \multirow{2}{*}{ Method } & \multicolumn{3}{|c|}{$P>-6^{\circ} \mathrm{C}$} & \multicolumn{3}{|c|}{$P>-7^{\circ} \mathrm{C}$} \\
\hline & $\mathrm{R}$ & Mean & RMS & $\mathrm{R}$ & Mean & RMS \\
\hline IDW & 0.9291 & 0.0059 & 0.1568 & 0.9164 & 0.0071 & 0.1612 \\
\hline GPI & 0.8357 & -0.0001 & 0.2323 & 0.8271 & -0.0001 & 0.2347 \\
\hline LPI & 0.9394 & -0.0004 & 0.1487 & 0.9302 & -0.0007 & 0.1528 \\
\hline $\mathrm{RBF}$ & 0.9663 & 0.0015 & 0.1515 & 0.9521 & 0.0024 & 0.1578 \\
\hline $\mathrm{OK}$ & 0.9666 & -0.0017 & 0.1509 & 0.9579 & 0.0026 & 0.1572 \\
\hline
\end{tabular}

Note: $P>-6^{\circ} \mathrm{C}$ or $P>-7^{\circ} \mathrm{C}$ has the same mean as Table 1 .

\subsubsection{Overwintering distribution predictions}

The overwintering distributions predicted from the modeling are shown in Fig. 3 and 4. Color changes from blue to yellow and then to red indicate the increase in the annual probability that the average temperature of the coldest month will be accompanied by increases in the probability of overwintering. The results show that the areas with high probability are mainly distributed in the winter wheat region in south-eastern China. The Pst overwintering boundary obtained in this study is broadly in line with what is generally considered to be the northern geographic boundary for Pst overwintering (the line from Dezhou through Shijiazhuang and Jiexiu to Huangling) [1], and the main difference was that the boundary in North China is closer to Beijing and was not limited to Dezhou, Shandong, similar to the results of Shi [19]. In this study, the number of selected historical overwintering locations distributed in the predicted overwintering areas of stripe rust was used to determine the critical probability suitable for Pst overwintering. As shown in Table 7, overall the number of the overwintering locations increased with the increase in the probability; specifically, the number increased greatly in areas where the probability was higher

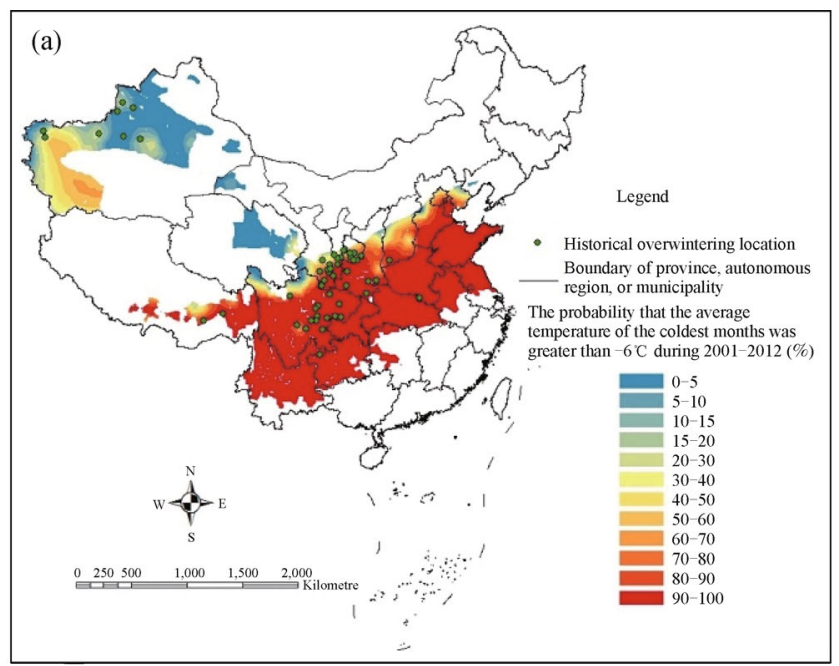

than $70 \%$, and this result is consistent with that obtained by Shi [19]. More than $70 \%$ of the selected historical overwintering locations were in areas with a probability higher than $70 \%$, and more than $50 \%$ of the selected historical overwintering locations were located in areas with a probability higher than $90 \%$. Therefore, the areas with a probability higher than $90 \%$ were regarded as areas suitable for Pst overwintering in China, the areas with a probability between $70 \%$ and $90 \%$ where there were approximately $20 \%$ of the selected historical overwintering locations were regarded as transitional zones, and the areas with a probability lower than $70 \%$, and where there were very few of the selected historical overwintering locations, were regarded as the areas that were not quite suitable for Pst overwintering.

4.2 Overwintering predictions based on the annual mean temperature of the coldest month and elevation model

Based on annual mean temperature of the coldest month and the elevation model, the ordinary Kriging interpolation results using the optimal interpolation method used in modeling the overwintering distribution of Pst are shown in Fig. 5 and 6. There were certain differences between the

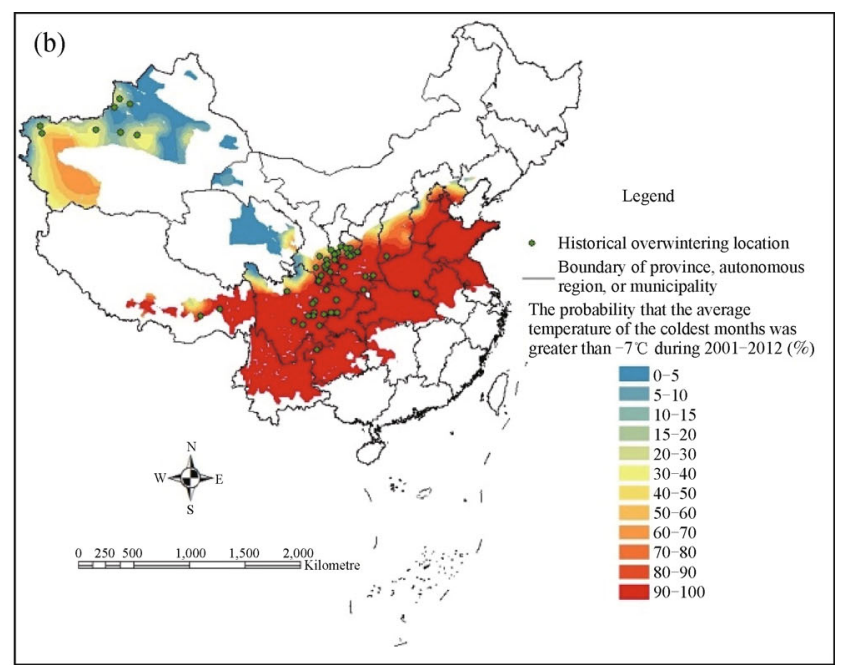

Fig. 3 Overwintering areas of stripe rust based on the annual probability of the critical temperature (exponential model). (a) The critical temperature was $-6^{\circ} \mathrm{C}$; (b) the critical temperature was $-7^{\circ} \mathrm{C}$. 

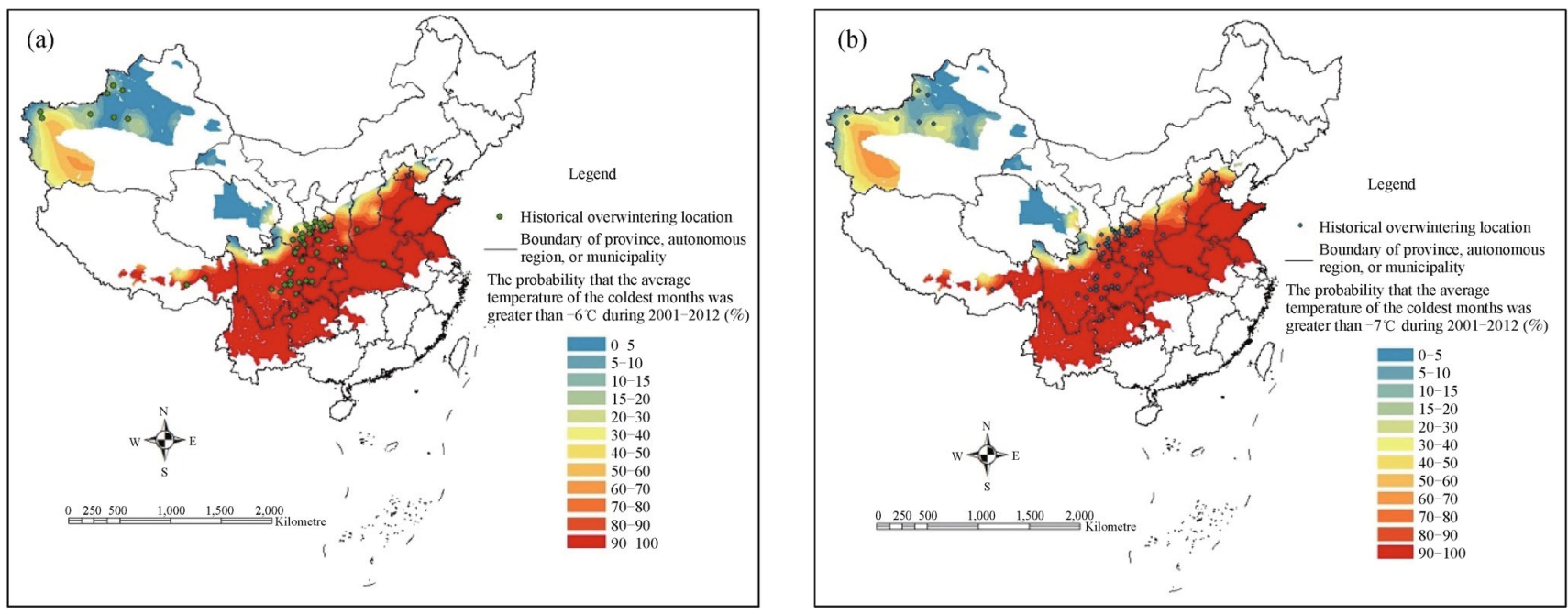

Fig. 4 Overwintering areas of stripe rust based on the annual probability of the critical temperature (spherical model). (a) The critical temperature was $-6^{\circ} \mathrm{C}$; (b) the critical temperature was $-7^{\circ} \mathrm{C}$.

Table 7 Statistics of selected historical oversummering locations in the predicted overwintering regions based on the annual probability using an exponential model and a spherical model

\begin{tabular}{|c|c|c|c|c|}
\hline \multirow{2}{*}{ Annual probability $/ \%$} & \multicolumn{2}{|c|}{ Exponential model } & \multicolumn{2}{|c|}{ Spherical model } \\
\hline & $P>-6^{\circ} \mathrm{C}$ & $P>-7^{\circ} \mathrm{C}$ & $P>-6^{\circ} \mathrm{C}$ & $P>-7^{\circ} \mathrm{C}$ \\
\hline$\overline{0-5}$ & 1 & 0 & 2 & 0 \\
\hline $5-10$ & 2 & 1 & 1 & 1 \\
\hline $10-15$ & 2 & 2 & 2 & 2 \\
\hline $15-20$ & 1 & 0 & 1 & 0 \\
\hline $20-30$ & 1 & 3 & 1 & 3 \\
\hline $30-40$ & 2 & 1 & 1 & 2 \\
\hline $40-50$ & 2 & 3 & 3 & 1 \\
\hline $50-60$ & 0 & 1 & 0 & 1 \\
\hline $60-70$ & 2 & 0 & 2 & 1 \\
\hline $70-80$ & 6 & 6 & 5 & 6 \\
\hline $80-90$ & 7 & 6 & 8 & 7 \\
\hline $90-100$ & 26 & 29 & 26 & 27 \\
\hline Total & 52 & 52 & 52 & 52 \\
\hline
\end{tabular}

Note: $P>-6^{\circ} \mathrm{C}$ or $P>-7^{\circ} \mathrm{C}$ has the same mean as Table 1 .

interpolation results obtained using the standard regression equation and the self-built regression equation, respectively. The results obtained using the same equation based on the exponential model and the spherical model coincided with one another. In consideration of the geographical distribution of historical overwintering locations and the phenomenon of global warming, overwintering distribution modeling was based on interpolation maps obtained using the self-built regression equation, and only the interpolation results obtained from the exponential model were analyzed.
The interpolation map obtained based on the exponential model was overlaid with the county level administrative map of China, local amplification of the overwintering predictions were obtained as shown in Figs. 7-10. Figures 7-10 demonstrated that the Pst overwintering boundary obtained is a line from Funing (Hebei) through Changping (Beijing), Pingshan (Hebei), Jiexiu (Shanxi), Ganquan (Shaanxi), Qingyang (Gansu), Tanchang (Gansu), Songpan (Sichuan), Aba (Sichuan), Luhuo (Sichuan), Shiqu (Sichuan) and Changdu (Tibet) to Linzhi (Tibet), and the winter wheat growing areas to the south of the line were 

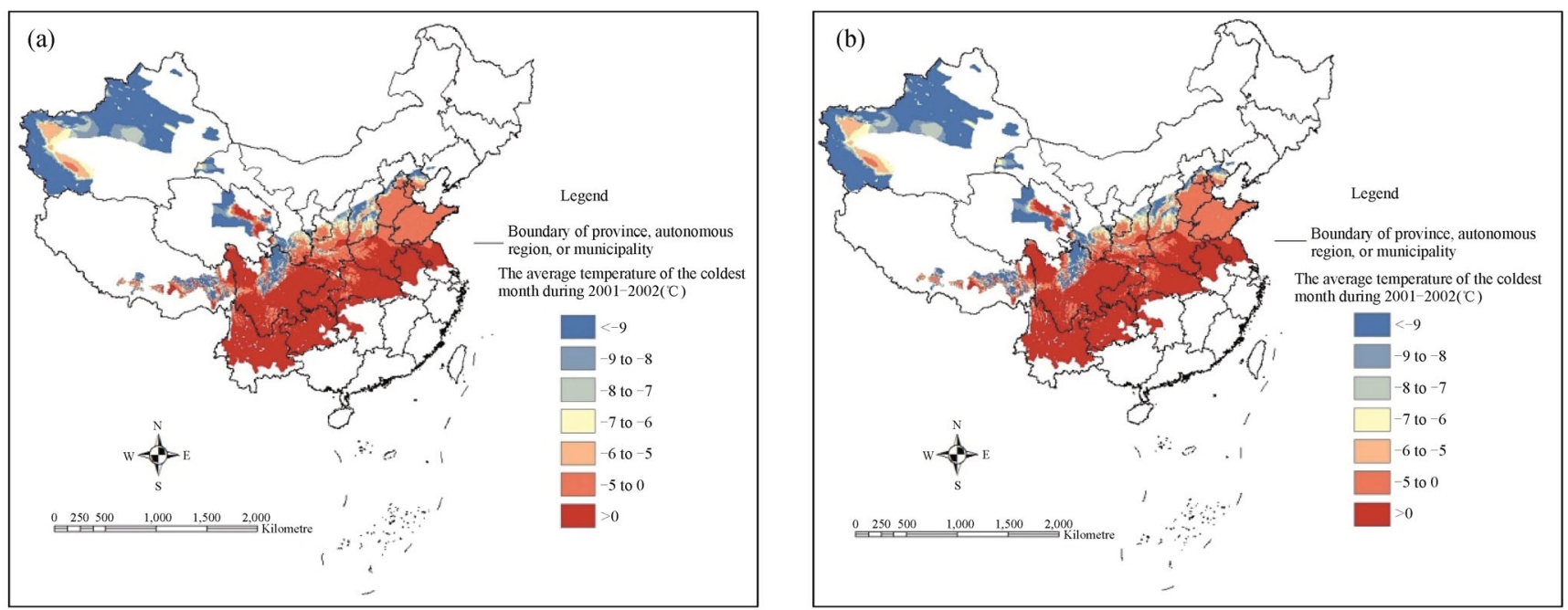

Fig. 5 Elevation interpolation using ordinary Kriging based on the standard regression equation. (a) Exponential model; (b) spherical model.
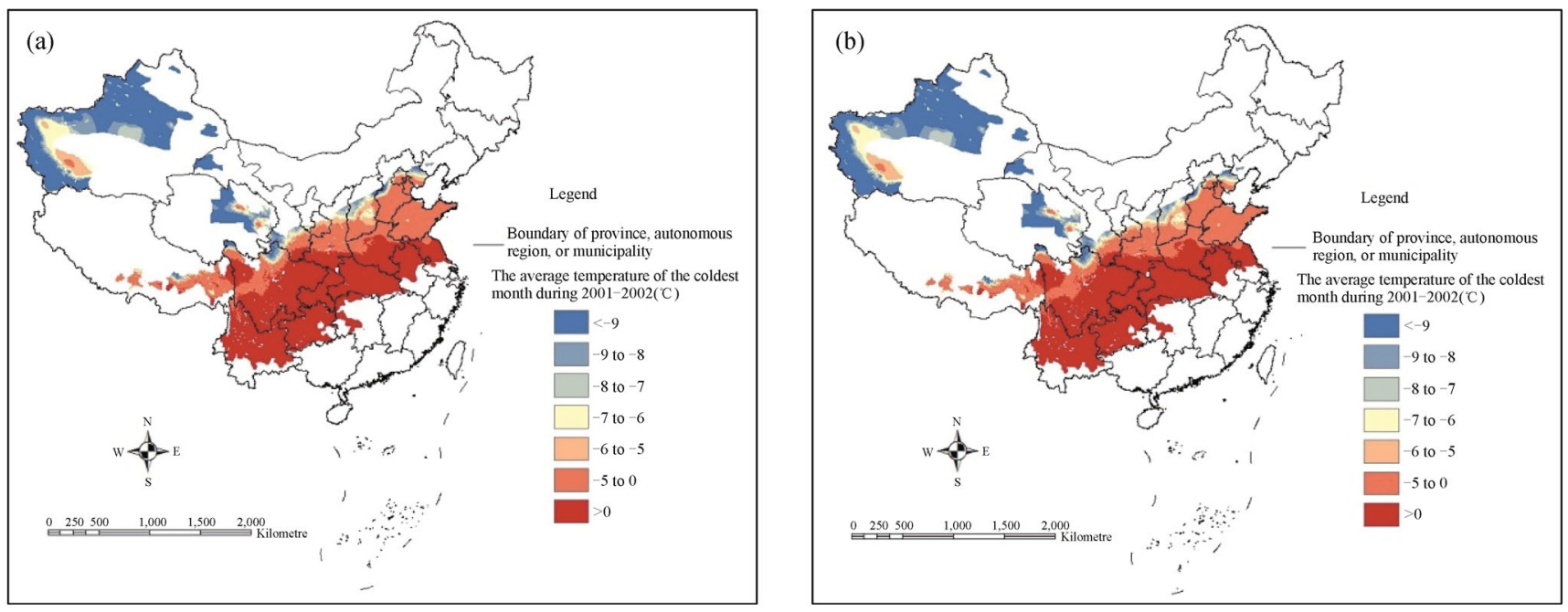

Fig. 6 Elevation interpolation using ordinary Kriging based on the self-built regression equation. (a) Exponential model; (b) spherical model.

predicted as suitable for Pst overwintering; however, the areas to the north of the line were predicted as marginal for the overwintering of Pst, and only a small part of these areas, including part of Wulan and Guinan in Qinghai Province, is suitable. In addition, the boundary for overwintering of Pst is consistent with the areas where the probability is $70 \%$ to $90 \%$. In the past, Shandong Province was generally considered to be the boundary in North China [1]. However, from our results the predicted boundary in North China was actually closer to Beijing, and this result was similar to that of Shi [19]. Moreover, as seen in Fig. 10, some regions in Xinjiang, including Akto, Pishan, Hotan and Yutian, were also predicted to be suitable for overwintering of stripe rust.

\section{Discussion}

Based on the probability that the average temperature of the coldest month during the period December, January and February from 2001 to 2012 in each meteorological station was higher than the critical temperature, modeling of overwintering of Pst was conducted using five interpolation methods including IDW, GPI, LPI, RBF and Kriging. The results showed that the ordinary Kriging (exponential model) was the optimal interpolation method. Interpolation to predict the distribution of Pst was conducted using the ordinary Kriging methods based on both the exponential model and the spherical model, and these results were compared with the overwintering 


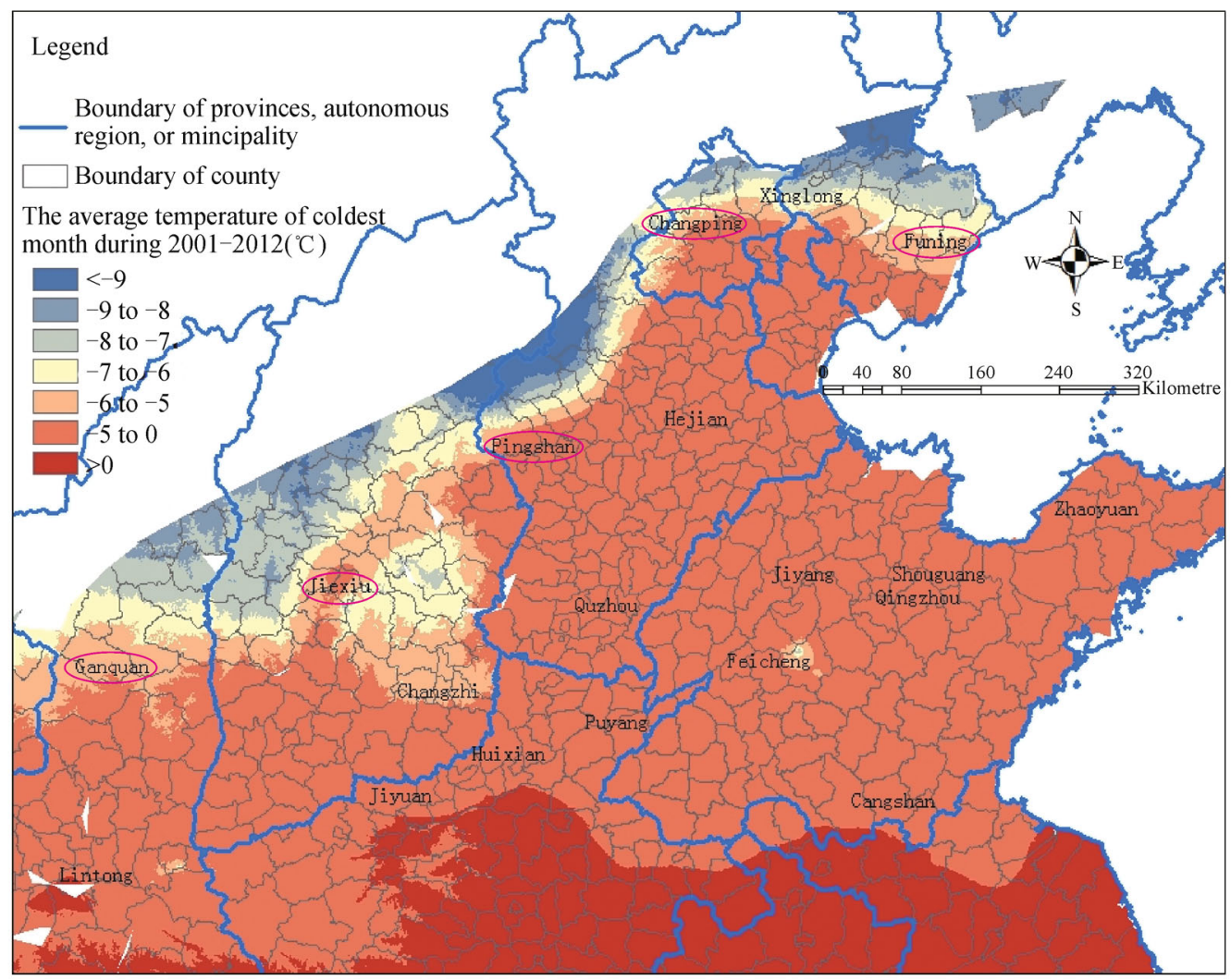

Fig. 7 Local amplification (a) of Fig. 6(a). The counties circled by pink line were on the boundary for Pst overwintering.

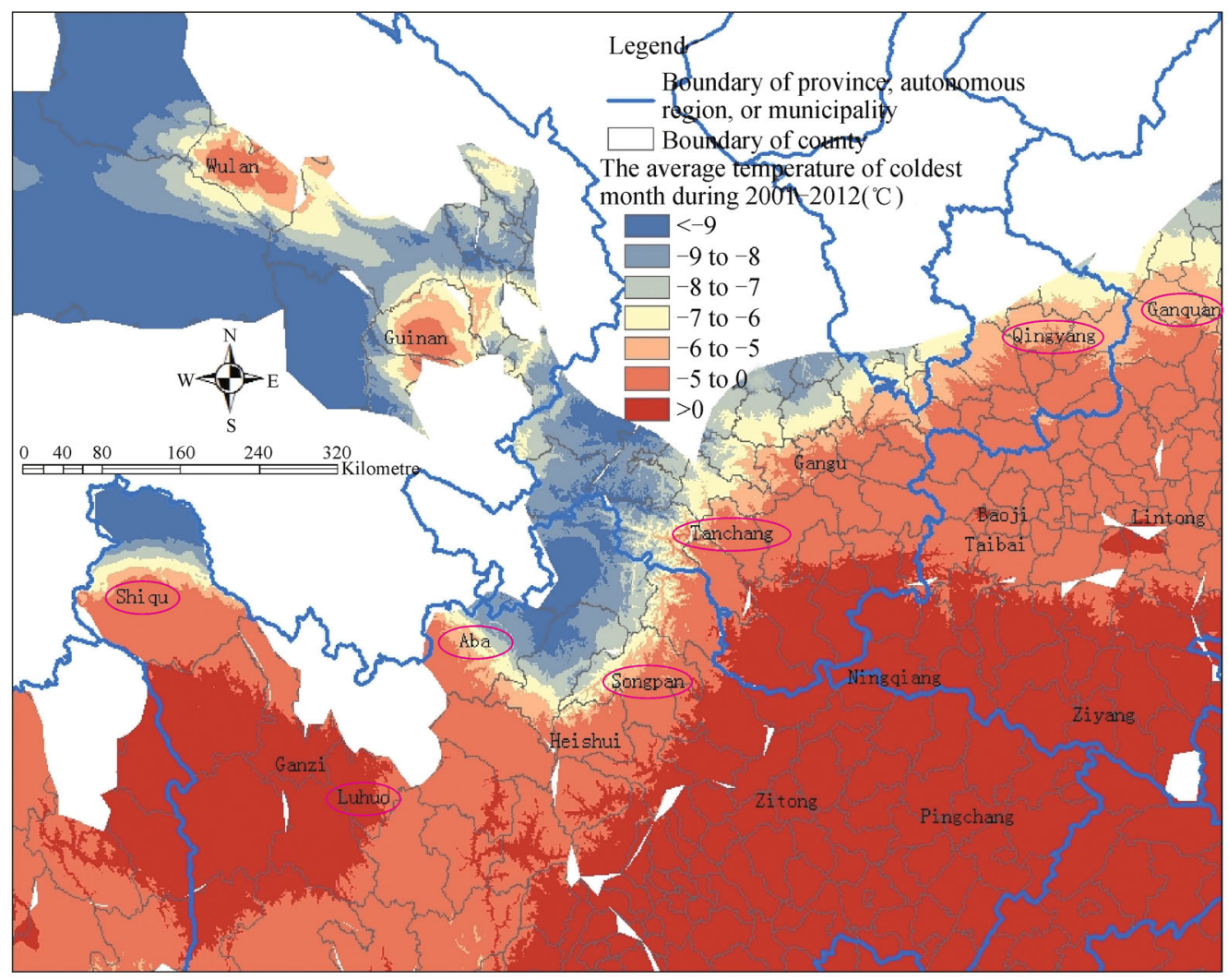

Fig. 8 Local amplification (b) of Fig. 6(a). The counties circled by pink line were on the boundary for Pst overwintering. 


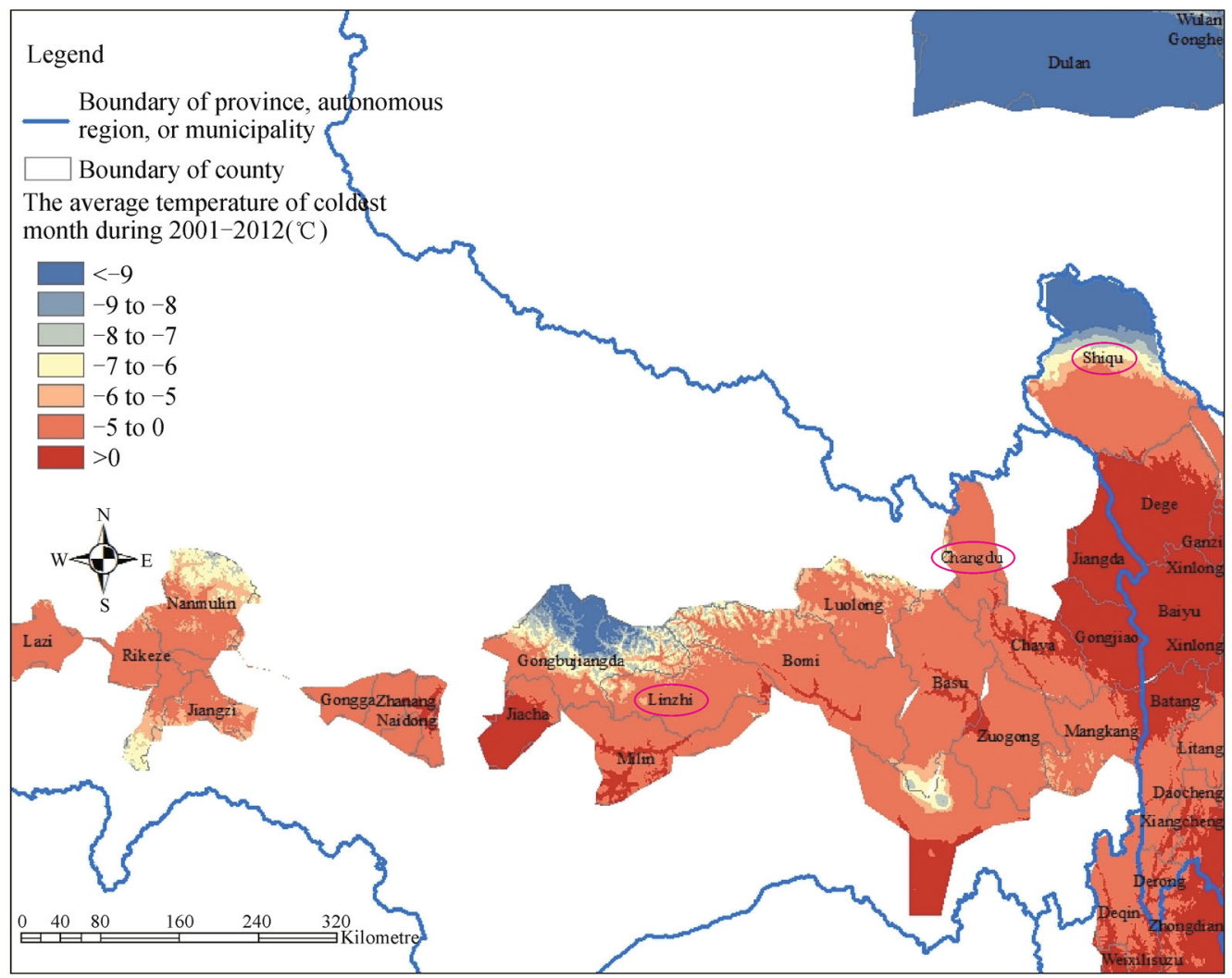

Fig. 9 Local amplification (c) in Fig. 6(a). The counties circled by pink line were on the boundary for Pst overwintering.

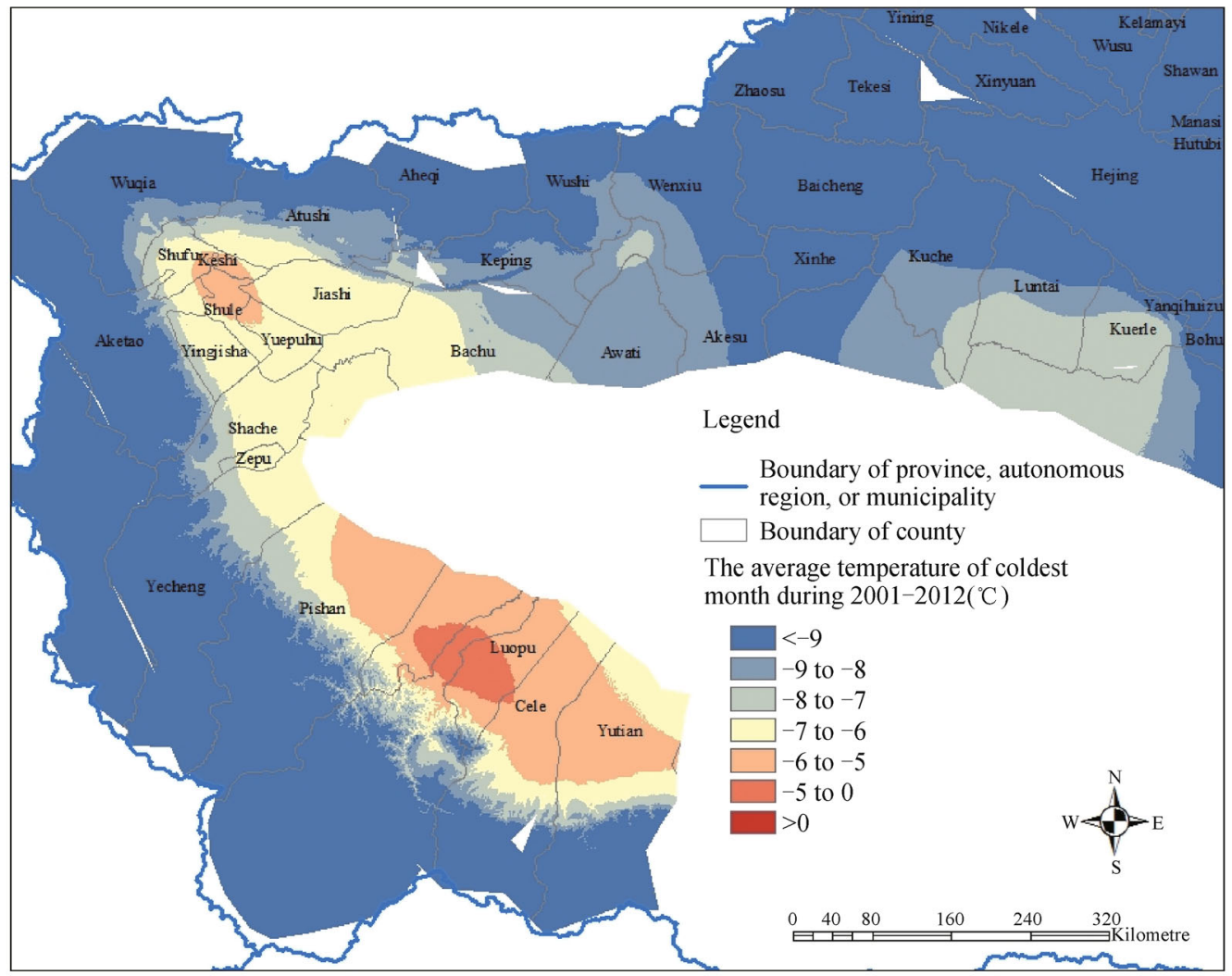

Fig. 10 Local amplification of Xinjiang in Fig. 6(a) 
distribution predictions of Shi [19], who used the ordinary Kriging (spherical model) based on the China meteorological data from 1960 to 2001. The winter wheat growing areas with an annual probability higher than $90 \%$ were regarded as areas suitable for Pst overwintering in China, the areas with a probability between $70 \%$ and $90 \%$ were regarded as transitional zones, and the areas with a probability lower than $70 \%$ were regarded as areas that were not quite suitable for Pst overwintering. However, only temperature was considered when the modeling of the overwintering distribution was conducted in this study, so the predictions should be further refined using other factors such as the amount of the oversummering inocula and the extent of snow cover. Moreover, when the modeling of overwintering distribution based on the probability was conducted, spatial factors such as longitude, latitude and altitude were not considered. Therefore, a more accurate method is needed for refining overwintering predictions for Pst.

Based on the annual average temperature of the coldest month in each year during the period December, January and February from 2001 to 2012, elevation-based interpolation was conducted using ordinary Kriging methods with the exponential model and the spherical model. There were no obvious differences between the results obtained using the ordinary Kriging methods based on the exponential model and the spherical model. The Pst overwintering boundary was obtained using this method. The winter wheat growing areas to the south of the line were suitable for Pst overwintering; however, the areas to the north of the line were rarely suitable for Pst overwintering, and only a small part of this area, including part of Wulan and Guinan in Qinghai Province, were suitable for Pst overwintering. This result is similar to that of Shi [19]. The boundary was extended in North China, northward to Beijing compared with the traditional boundary. This may be related to global climate warming. Some areas in Xinjiang, including Akto, Pishan, Hotan and Yutian, were also found to be suitable for overwintering of stripe rust. However, only the regression relationship between altitude and temperature was considered in the elevation-based interpolation in this study. The overwintering predictions can be optimized if the raster data of the latitude and longitude of each meteorological station were utilized.

Due to the lack of meteorological data on snow cover at each meteorological station during the years 2001 to 2012, the influence of snow on the overwintering of Pst was not considered in the study. Pst in wheat seedlings covered by snow in the field can overwinter safely even when the air temperature is lower than $-10^{\circ} \mathrm{C}$; in fact, some areas with snow cover in China may become overwintering areas for Pst, and the ranges for Pst overwintering may have expanded somewhat. This poissibility needs further attention, taking into account the specific meteorological conditions.
Frequent extreme weather in China will affect the epidemics of stripe rust. Especially in recent years, long drought or extremely dry weather has often occurred in the important areas for stripe rust in south-western and northwestern China. These types of weather will affect overwintering of Pst, so further research is needed. In addition, because limited data were available, the effects of factors such as climatic change on wheat cultivation and wheat growing areas in China were not considered in this study. The relevant data will also be needed to optimize the models.

\section{Conclusions}

The overwintering predictions obtained in this study still need to be analyzed comprehensively in combination with the actual prevalence of the disease on the autumn seedlings, wheat cultivation, frost resistance of wheat, and specific meteorological conditions. Nevertheless, these results can provide the basis and support for monitoring and forecast of stripe rust and for prevention and control of the spring epidemics of the disease.

Acknowledgements This study was supported by the National Natural Science Foundation of China (31101393) and the National Key Technologies Research and Development Program (2012BAD19BA04).

Compliance with ethics guidelines Xiaojing Wang, Zhanhong Ma, Yuying Jiang, Shouding Shi, Wancai Liu, Juan Zeng, Zhiwei Zhao and Haiguang Wang declare that they have no conflict of interest or financial conflicts to disclose.

This article does not contain any studies with human or animal subjects performed by any of the authors.

\section{References}

1. Li Z Q, Zeng S M. Wheat rusts in China. Beijing: China Agriculture Press, 2002, 1-254 (in Chinese)

2. Wan A M, Chen X M, He Z H. Wheat stripe rust in China. Australian Journal of Agricultural Research, 2007, 58(6): 605-619

3. Chen W Q, Kang Z S, Ma Z H, Xu S C, Jin S L, Jiang Y Y. Integrated management of wheat stripe rust caused by Puccinia striiformis f. sp. tritici in China. Scientia Agricultura Sinica, 2013, 46(20): 4254-4262 (in Chinese)

4. Wang H G, Yang X B, Ma Z H. Investigation of the long-distance transports of Puccinia striiformis f. sp. tritici in China using HYSPLIT-4 model. Journal of China Agricultural University, 2010, 15(5): 55-64 (in Chinese)

5. Qin D H, Ding Y H, Su J L, Ren J W, Wang S W, Wu R S, Yang X Q, Wang S M, Liu S Y, Dong G R, Lu Q, Huang Z G, Du B L, Luo Y. Assessment of climate and environment changes in China (I): climate and environment changes in China and their projection. Advances in Climate Change Research, 2005, 1(1): 4-9 (in Chinese)

6. Chakraborty S, Tiedemann AV, Teng P S. Climate change: potential 
impact on plant diseases. Environmental Pollution, 2000, 108(3): 317-326

7. Runion G B. Climate change and plant pathosystems - future disease prevention starts here. New Phytologist, 2003, 159(3): 531533

8. Mcelrone A J, Reid C D, Hoye K A, Hart E, Jackson R B. Elevated $\mathrm{CO}_{2}$ reduces disease incidence and severity of a red maple fungal pathogen via changes in host physiology and leaf chemistry. Global Change Biology, 2005, 11(10): 1828-1836

9. Madgwick J W, West J S, White R P, Semenov M A, Townsend J A, Turner J A, Fitt B D L. Impacts of climate change on wheat anthesis and fusarium ear blight in the UK. European Journal of Plant Pathology, 2011, 130(1): 117-131

10. Li Y J, Wang C Y, Zhao B, Liu W J. Effects of climate change on agricultural meteorological disaster and crop insects diseases. Transactions of the CSAE, 2010, 26(Supp.1): 263-271 (in Chinese)

11. Huo Z G, Li M S, Wang L, Wen Q P, Xiao J J, Huang D P, Wang C $\mathrm{Y}$. Impacts of climate warming on crop diseases and pests in China. Scientia Agricultura Sinica, 2012, 45(10): 1926-1934 (in Chinese)

12. Huo Z G, Li M S, Li N, Wang L, Huang D P, Wang C Y. Impacts of seasonal climate warming on crop diseases and pests in China. Scientia Agricultura Sinica, 2012, 45(11): 2168-2179 (in Chinese)

13. Chakraborty S, Luck J, Hollaway G, Fitzgerald G, White N. Rustproofing wheat for a changing climate. Euphytica, 2011, 179(1): 1932

14. Juroszek P, von Tiedemann A. Climate change and potential future risks through wheat diseases: a review. European Journal of Plant Pathology, 2013, 136(1): 21-33
15. Nelson M R, Orum T V, Jaime-Garcia R, Nadeem A. Applications of geographic information systems and geostatistics in plant disease epidemiology and management. Plant Disease, 1999, 83(4): 308319

16. Jaime-Garcia R, Orum T V, Felix-Gastelum R, Trinidad-Correa R, Vanetten H D, Nelson M R. Spatial analysis of Phytophthora infestans genotypes and late blight severity on tomato and potato in the Del Fuerte Valley using geostatistics and geographic information systems. Phytopathology, 2001, 91(12): 1156-1165

17. Chen C, Chen J, Hu B S, Bai Y Z, Jiang Y H, Liu F Q. Potential distribution of Erwinia amylovora in China and invasion risk assessment. Scientia Agricultura Sinica, 2007, 40(5): 940-947 (in Chinese)

18. Li B, Cao X, Chen L, Zhou Y, Duan X, Luo Y, Fitt B D L, Xu X, Song Y, Wang B, Cao S. Application of geographic information systems to identify the oversummering regions of Blumeria graminis f. sp. tritici in China. Plant Disease, 2013, 97(9): 11681174

19. Shi S D. Climate-based regional classification for oversummering and overwintering of Puccinia striiformis and spatio-temporal dynamic analysis in China with GIS. Dissertation for the Master Degree. Beijing: China Agricultural University, 2004 (in Chinese)

20. China's national Atlas compilation committee. National agricultural Atlas of the People's Republic of China. Beijing: SinoMaps Press, 1989, 1-161 (in Chinese)

21. Cui D C, Cao G C, Zhang W, Zhang C Q. Climat-ecological regionalization of wheat in China. Guizhou: Guizhou Science and Technology Press, 1991, 1-97 (in Chinese) 